\title{
Upregulation of MicroRNA-210 Regulates Renal Angiogenesis Mediated by Activation of VEGF Signaling Pathway under Ischemia/Perfusion Injury in vivo and in vitro
}

\author{
Fen Liu ${ }^{a}$ c Yuan-Lei Lou ${ }^{a}$ Jue Wu ${ }^{a}$ Qiong-Fang Ruan ${ }^{a} \quad$ An Xie $^{a}$ Fei Guo \\ Su-Ping Cui ${ }^{a}$ Zhi-Feng Deng ${ }^{b}$ Yang Wang ${ }^{a, d}$ \\ Institutes of a Urology and ${ }^{\mathrm{b} C}$ Cerebrovascular Diseases, Nanchang University, and ' Intensive Care Unit, \\ First Affiliated Hospital of Nanchang University, Nanchang, and d Institute of Orthopedic Surgery, Shanghai Sixth \\ People's Hospital, Shanghai Jiao-tong University, Shanghai, China
}

\section{Key Words}

MicroRNA $\cdot$ Angiogenesis $\cdot$ Ischemia/reperfusion

\begin{abstract}
Background: MicroRNAs (miRNAs) are endogenous, noncoding, small RNAs that regulate gene expression and function, but little is known about regulation of miRNAs in the kidneys under normal or pathologic conditions. Here, we sought to investigate the potential involvement of miRNAs in renal ischemia/reperfusion (I/R) injury and angiogenesis and to define some of the miRNAs possibly associated with renal angiogenesis. Methods and Results: Male Balb/c mice were subjected to a standard renal I/R. CD31 immunostaining indicated a significant increase of microvessels in the ischemic region. VEGF and VEGFR2 expression were increased in renal I/R at both the mRNA and protein levels which were detected by qRT-PCR and Western blot, respectively. More importantly, 76 microRNAs exhibited more than 2-fold changes using Agilent microRNA microarray, which
\end{abstract}

F. Liu and Y.-L. Lou contributed equally to this work.

\section{KARGER}

Fax +41613061234 E-Mail karger@karger.ch www.karger.com
(C) 2011 S. Karger AG, Base

$1420-4096 / 12 / 0353-0182 \$ 38.00 / 0$

Accessible online at:

www.karger.com/kbr contains downregulation of 40 miRNAs and upregulation of 36 miRNAs. Upregulation of miR-210 was confirmed by qRTPCR with prominent changes at 4 and $24 \mathrm{~h}$ after reperfusion. Furthermore, overexpression of miR-210 in HUVEC-12 cells enhances VEGF and VEGFR2 expression and promotes angiogenesis on Matrigel in vitro. Conclusion: These findings suggest miR-210 may be involved in targeting the VEGF signaling pathway to regulate angiogenesis after renal I/R injury, which provides novel insights into the angiogenesis mechanism of renal I/R injury.

Copyright $\odot 2011$ S. Karger AG, Basel

\section{Introduction}

A large body of evidence indicates that ischemia/reperfusion (I/R) injury results in dysfunctions of organs, such as kidney [1], liver [2], intestine [3] and so forth. In particular, renal I/R injury is a common cause of acute kidney injury in several settings including renal transplantation. 
Several mediators and pathways have been proposed to be involved in the injury. Among them, a major physiological response to ischemia is neovascularization, which involves a sequence of events resulting in development of new capillaries from endothelial cells or preexisting vessels.

In recent decades, extensive studies have revealed a variety of angiogenic factors and their receptors, including vascular endothelial growth factor (VEGF)-VEGFRs, Angiopoietin-Tie, Ephrin-EphRs and Delta-Notch to be the most important regulators of angiogenesis in vertebrates [4]. Vincent et al. [5] showed that overexpression of HIF-1 $\alpha /$ VP16 in HeLa, C6 and Hep3B cells led to upregulation of VEGF, and in an in vivo study they proved that treatment with HIF-1 $\alpha /$ VP16 or VEGF in a model of hindlimb ischemia improved angiographic score and capillary density. These findings indicate that the VEGF signaling pathway may be the most potent stimulator of angiogenesis [6]. However, the precise protective mechanisms involved in renal ischemic injury and angiogenesis remain to be determined.

Other reports displayed that miRNAs also play an important role during angiogenesis after IR injury [7]. First, evidence of miRNAs involvement in the regulation of angiogenesis was found by the inhibition of Dicer and Drosha, two key nucleases involved in miRNA maturation [8]. More interestingly, selective manipulation of a specific miRNA has been correlated with angiogenesis processes in ischemic diseases [9]. Knockdown of miR-126 in zebrafish resulted in loss of vascular integrity and hemorrhage during embryonic development [10]. miRNAs are a family of endogenous non-coding RNAs of 22-24 nucleotides, which have emerged as important regulators of various physiologic and pathologic processes in eukaryotes [11], such as development, differentiation, metabolism, growth, proliferation and apoptosis [12]. Deregulations of miRNAs are associated with various diseases [13] and microarray analysis on the expression profiles of microRNAs changes also were performed on the acute ischemic tissue, including myocardium [14], hippocampi [15], liver [16], etc. Notably, miR-210 has been found to be upregulated in response to brain transient focal ischemia in rats [17]. These studies provide strong evidence that miRNAs play important regulatory roles in ischemic diseases.

Our question is whether renal miRNAs also play an important regulatory role in ischemia. To address this issue, we employed miRNA microarray to investigate potential involvement of microRNAs in mice kidneys subjected to I/R injury, and to identify some of the micro RNAs possibly associated with renal angiogenesis. Our data show that VEGF signaling pathway and miRNAs were indeed involved in angiogenesis after renal I/ $\mathrm{R}$ injury, and these studies provide potential mechanisms by which miRNA-210 regulates VEGF and VEGFR2 expression in vitro.

\section{Materials and Methods}

\section{Renal I/R Injury}

Studies were performed on male Balb/c mice (7-8 weeks old) that were purchased from Nanchang University (Nanchang, China). Renal I/R was induced in mice as described previously [18, 19]. All animal procedures were conducted after approval from the Animal Committee of Nanchang University. Briefly, mice were intraperitoneally anesthetized with $50-60 \mathrm{mg} / \mathrm{kg}$ pentobarbital sodium and kept on a homoeothermic station to maintain body temperature at $37^{\circ} \mathrm{C}$. Kidneys were exposed by bilateral flank incision, and the renal pedicles were clamped to induce ischemia. After ischemia, the arterial clamps were released for reperfusion. Sham control animals were subjected to identical operation without renal pedicle clamping.

Mice were allowed to recover for 4, 24 and $48 \mathrm{~h}$ after ischemia or sham surgery. The kidneys were removed at indicated time points, cut in half longitudinally and snap-frozen in liquid nitrogen or stored at $-80^{\circ} \mathrm{C}$ for subsequent biochemical analysis. In the sham group, all samples were collected at $24 \mathrm{~h}$ after sham surgery.

Measurement of Serum Biochemical Parameters

The serum creatinine (SCR) and urea nitrogen (BUN) levels were measured as a marker of kidney injury using a 7180 Automatic Analyzer (Hitachi, Japan) according to the manufacturer's instructions.

\section{Immunohistochemistry Staining}

In order to examine ischemia-induced angiogenesis in renal ischemia, we performed immunohistochemistry staining to detect the endothelial cell marker CD31 expression. Tissues for light microscopy were fixed in $4 \%$ paraformaldehyde, embedded in paraffin, and cut into $4-\mu \mathrm{m}$ sections for immunoperoxidase staining as described elsewhere $[20,21]$. Briefly, to perform immunoperoxidase staining, tissue sections were incubated with the following primary and secondary antibodies as indicated. Primary antibody was rat polyclonal anti-mouse CD31 antibody (Chemicon, Temecula, Calif., USA); secondary antibody was horseradish peroxidase (HRP)-conjugated goat anti-rat IgG (Sigma, St. Louis, Mo., USA). Negative controls for immunostaining included either deletion of the primary antibody or substitution of the primary antibody with equivalent concentrations of an irrelevant murine or rat $\mathrm{mAb}$. All tissue sections were incubated with primary antibodies overnight at $4^{\circ} \mathrm{C}$. Afterwards, specific biotinylated secondary antibodies were applied followed by peroxidase-conjugated Avidin D. Angiogenesis was assessed semiquantitatively after immunohistochemistry staining for CD31-positive cells on sections using a $10 \times 10$ eyepiece grid to count [22].

Histological Examination of Kidney

To corroborate the functional analysis, tissue samples were fixed in $4 \%$ paraformaldehyde, embedded in paraffin, sectioned 
at $4 \mu \mathrm{m}$ thickness, and stained with hematoxylin and eosin (HE). Light microscopy was used to observe the degree of renal damage.

\section{Cell Lines}

HUVEC-12 cells were maintained in a Dulbecco's modified Eagle's minimum supplemented with $10 \%$ fetal bovine serum, penicillin $(100 \mathrm{U} / \mathrm{ml})$ and streptomycin $(100 \mu \mathrm{g} / \mathrm{ml})$, in a humidified incubator $\left(95 \%\right.$ air $\left./ 5 \% \mathrm{CO}_{2}\right)$ at $37^{\circ} \mathrm{C}$. HUVEC- 12 cells were infected twice with the viral particles containing LV-miR-210GFP or LV-miR-GFP vector as described below. The expression of miR-210 in cells was confirmed using real-time PCR and GFP expression observed under a fluorescence microscope at $72 \mathrm{~h}$ after infection.

For angiogenesis experiments, HUVEC-12 cells expressing miR-210 or control were cultured on Matrigel in 96-well plates. $24 \mathrm{~h}$ later, tube-like structures were randomly counted in 5 different fields using $10 \times$ magnifications.

\section{Lentivirus Production}

Lentivirus was produced after transient transfection of human embryonic kidney (HEK) 293T cells with LV-miR-210-GFP or LVmiR-GFP vector along with the packaging plasmids pCMV-VSV$\mathrm{G}$ and psPAX2 (Addgene) using FuGEN6-HD (Roche Diagnostics, Mannheim, Germany) as the transfection reagent according to Roche's protocol. The supernatants containing viral particles were collected $48 \mathrm{~h}$ after the transfection, filtered through a 0.22 $\mu \mathrm{m}$ filter and used to infect the HUVEC-12 cell line.

\section{Array Hybridization and Data Analysis}

To assess the level and composition of miRNA, microarrays from Agilent Technologies were used. Total RNA, including mi RNA, was extracted from $100 \mathrm{mg}$ of tissue by using the mirVana total RNA extraction kit following the instruction (Applied Biosystems/Ambion). The quality and quantity of the RNA was evaluated by 260/280 ratio and Agilent 2100 Bioanalyzer (Agilent Technologies). Subsequently, $100 \mathrm{ng}$ of total RNA was dephosphorylated and ligated with pCp-Cy3. Labeled RNA was purified and hybridized to Agilent miRNA arrays (v12.0). Images were scanned with the Agilent microarray scanner (Agilent), gridded, and analyzed using Agilent feature extraction software version 9.5.3.

\section{Real-Time Quantification of MicroRNAs by Stem-Loop}

RT-PCR

To validate the microarray results and identify some of the microRNAs associated with renal angiogenesis, quantitative realtime PCR was performed to examine miR-210 expression of different groups in renal I/R injury according to the operator's manual [23]. All primers used in this study were designed with Primer Express 3.0 software. Total RNA was extracted and reverse transcribed to cDNA with stem-loop primer and was amplified by SYBR Green real-time PCR using microRNA-specific primers in optimal reaction system. For reverse transcription, $100 \mathrm{ng}$ total RNA was diluted in a $20-\mu l$ reaction mix and reverse transcribed under the following conditions: $16^{\circ} \mathrm{C}$ for $30 \mathrm{~min}$, followed by RT of 60 cycles at $30^{\circ} \mathrm{C}$ for $30 \mathrm{~s}, 42^{\circ} \mathrm{C}$ for $30 \mathrm{~s}$ and $50^{\circ} \mathrm{C}$ for $1 \mathrm{~s}$; then incubated at $70^{\circ} \mathrm{C}$ for $15 \mathrm{~min}$. For real-time PCR amplification, $20 \mu \mathrm{l}$ of mix included $1 \mu \mathrm{l}$ reverse transcription product, $1 \mu \mathrm{l}$ miR-X $(2 \mathrm{mM}), 1 \mu \mathrm{l}$ universal primer $(2 \mathrm{mM})$, and $0.5 \mu \mathrm{l} 10 \times$
SYBR Green I were reacted on a Real-Time PCR System (ABI7500) as follows: $95^{\circ} \mathrm{C}$ for $10 \mathrm{~min}$ followed by 40 cycles of $95^{\circ} \mathrm{C}$ for $15 \mathrm{~s}$, and $60^{\circ} \mathrm{C}$ for $60 \mathrm{~s}$. All reactions were run in triplicate for each mouse, and the result for each mouse is the average of the triplicate. The data were normalized to U6. The threshold cycle (Ct) is defined as the fractional cycle number at which the fluorescence passes the fixed threshold. The miRNA relative expression level was calculated using the comparative Ct method $\left(2^{-\Delta \Delta C t}\right)$.

\section{Real-Time Quantification RT-PCR of $m R N A$}

Quantitative real-time PCR was performed to examine VEGF and VEGFR2 mRNA expression changes of different groups in the kidneys of mice with I/R injury. Total RNA was extracted from tissues using TRIzol (Sigma). Whole tissue samples were homogenized in TRIzol using a homogenizer. Sample RNA levels were evaluated by $260 / 280$ ratio. Total RNA was transcribed to cDNA with random primer and was amplified by SYBR Green real-time PCR using VEGF- and VEGFR2-specific primers in optimal reaction system. The data were normalized to GAPDH and relative expression was calculated using the comparative $\mathrm{Ct}$ method $\left(2^{-\Delta \Delta C t}\right)$

\section{Western Blot Analysis}

Western Blot was performed to detect VEGFR2 protein expression changes of different groups in the kidneys of mice with I/R injury. Proteins from tissues were resolved by SDS-PAGE and immunoblotting. Briefly, $50 \mu \mathrm{g}$ total protein was separated by $12 \%$ SDS-PAGE and transferred to nitrocellulose membranes using a trans-blot apparatus. After it was blocked in Tris-buffered saline (TBS) containing $0.1 \%$ Tween 20 (TBS-T) and 5\% non-fat dried milk (blocking buffer) at room temperature for $2 \mathrm{~h}$, the membranes were incubated with a primary antibody diluted in blocking buffer $(1: 200)$ at $4^{\circ} \mathrm{C}$ overnight. Next, the membranes were incubated with a secondary HRP-conjugated antibody diluted in blocking buffer $(1: 2,000)$ at room temperature for $1 \mathrm{~h}$ and were then washed 5 times in TBS-T. After successive washes, the membranes were developed with an enhanced chemiluminescence kit, and the results were recorded by X-ray films. Protein bands were visualized using a chemiluminescence reagent (Pierce Biotechnology, Rockford, Ill., USA) and then developed on Biomax Light Film (Kodak, Shanghai, China). The primary antibody used was rabbit polyclonal anti-mouse VEGFR2 antibody dilution (1:200 dilution; Santa Cruz Biotechnology, Inc., Santa Cruz, Calif., USA). The secondary antibody was HRP-conjugated goat anti-rabbit IgG (1:2,000 dilution; Sigma). $\beta$-Actin was used as a loading control. All Western blot experiments were repeated at least 3 times.

Statistical Analysis

Data are expressed as mean \pm SD. Data were analyzed using SPSS version 10.0. One-way ANOVA was used for comparisons of the data. Differences were considered significant when $\mathrm{p}<0.05$.

\section{Results}

\section{Establishment of Ischemic Injury Model}

In order to study effects of renal ischemic injury, we used an I/R injury model as our working model. First, we 
Fig. 1. Changes of biochemistry and histopathology in kidney after I/R. a, b SCR and BUN levels after I/R injury. Male Balb/c mice that underwent bilateral clamping of the renal pedicles for 45 min developed renal ischemic injury with a peak in SCR and BUN levels after I/R $24 \mathrm{~h}$. c HE staining showed that $24 \mathrm{~h}$ following ischemic injury caused significant damage (arrows in right panel), which manifested primarily as cytoplasmic vacuolization, cell necrosis of the proximal convoluted tubule, and tubular lumen obstruction and impairments (upper panel $\times 200$, lower panel $\times 400$ ). ${ }^{*} \mathrm{p}<0.05$ vs. sham control.

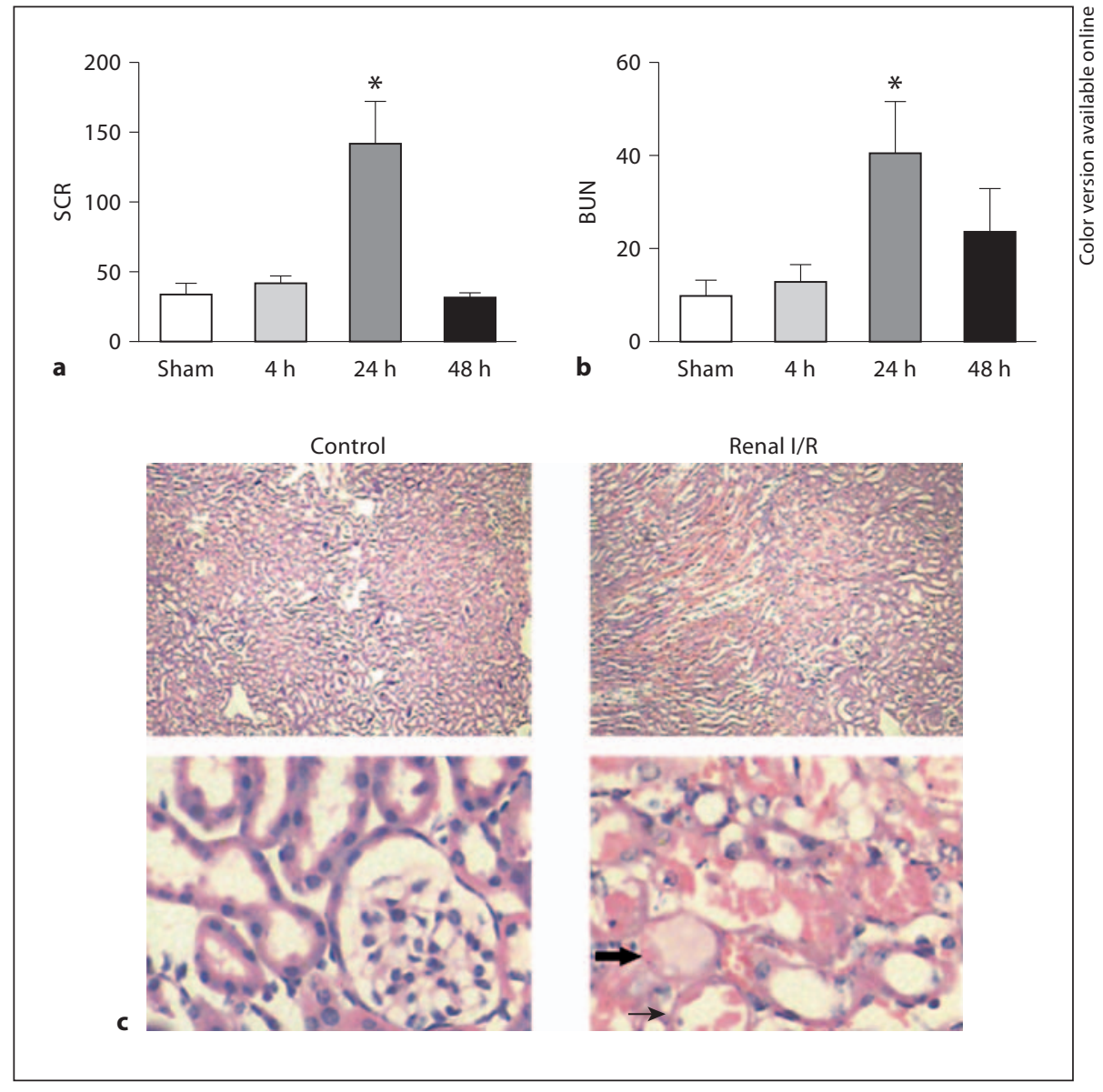

had to successfully establish a mouse model of renal I/R injury. To do so, we measured serum levels of both SCR and BUN, which are surrogate markers for renal function after renal ischemic injury. As shown in figure la and b, serum SCR and BUN levels were comparable to the shamoperated group at $24 \mathrm{~h}$ after bilateral clamping of the renal pedicles for $45 \mathrm{~min}$. In contrast, the SCR level was significantly increased $(142.4 \pm 29.42 \mu \mathrm{mol} / \mathrm{l})$ in the mice subjected to I/R injury compared to sham-operated mice $(34.02 \pm 7.06 \mu \mathrm{mol} / \mathrm{l})$ at $24 \mathrm{~h}$ following ischemia. The BUN level also followed the same trend as SCR, from $9.9 \pm 3.3 \mathrm{mmol} / \mathrm{l}$ in sham-operated animals to $40.5 \pm$ $11.03 \mathrm{mmol} / \mathrm{l}$ at $24 \mathrm{~h}$ after ischemia.

To further evaluate the severity of ischemic injury, we removed the kidneys and performed a pathological observation. Figure 1c shows that renal tissue suffered significant damage in mice at $24 \mathrm{~h}$ after ischemic injury. In accordance with a previous study, HE staining showed that there was significant damage at $24 \mathrm{~h}$ following ischemic injury, which manifested primarily as cytoplasmic vacuolization, cell necrosis of the proximal convoluted tubule, and tubular lumen obstruction and impairments. In contrast, no sign of ischemic damage was observed in the sham-operated mice, which is consistent with serum SCR and BUN levels of kidney ischemic injury. Taken together, we confirmed that the renal I/R injury model succeeded based on biochemistry measurement and pathological level.

\section{Angiogenesis in Response to Ischemic Injury}

Because neovascularization is a major physiological response to I/R injury, we examined ischemia-induced angiogenesis in renal ischemia on the capillary changes, and quantified renal microvascular density following the ischemic insult in our mouse model of renal I/R. We utilized the endothelial cell marker CD31 to identify renal angiogenesis by immunohistochemistry staining. There is a large quantity of CD31-positive cells in the endothelial layer at 24 and $48 \mathrm{~h}$ after I/R injury compared to the sham group (fig. 2a, b). We used the stereological method 
Fig. 2. Ischemia-induced angiogenesis in kidney. a, b CD31 immunohistochemistry staining. Renal tissues were taken out at 24 and $48 \mathrm{~h}$ after I/R injury, respectively. In the sham group, kidneys were collected at $24 \mathrm{~h}$ after sham manipulation. CD31 was used as a marker for microvessels. The number of positive cells at 24 and $48 \mathrm{~h}$ is higher than that in the sham control group. The arrows indicate CD31-positive staining. a Magnification $\times 200$, b magnification $\times 400$. c The stereology method was used to count the number of microvessels per field. A significant increment of neovascularization in ischemic region in renal ischemia compared with control group. ${ }^{*} \mathrm{p}<0.05$ vs. sham control.

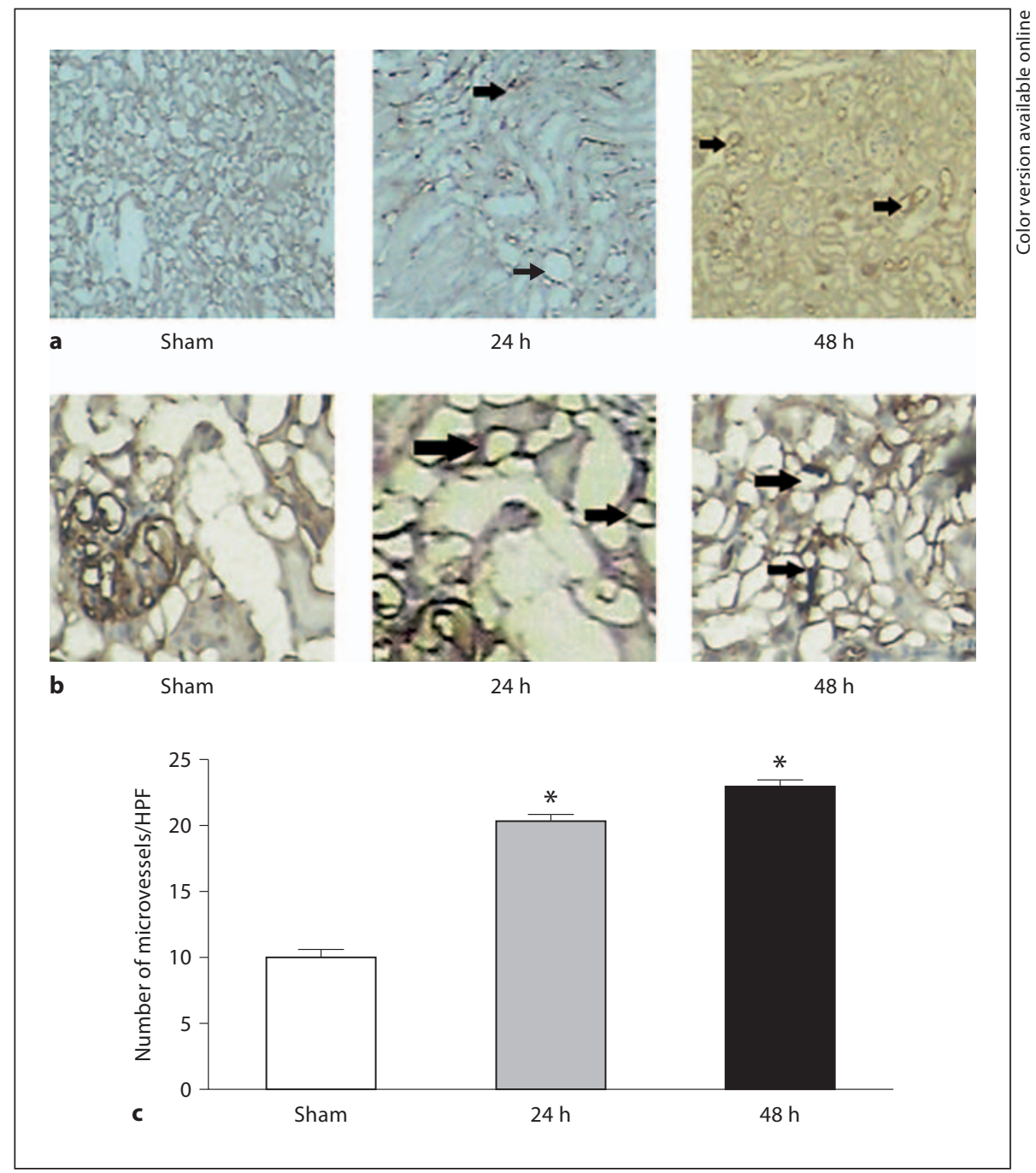

to analyze the number of microvessels and found a significant increase of neovascularization in the ischemic region in renal ischemia compared to the control group regardless of 24 or $48 \mathrm{~h}$ after I/R injury (fig. 2c; p < 0.05), indicating that angiogenesis indeed appeared in the kidneys after I/R injury.

\section{VEGF and VEGFR2 Expression Was Changed after Ischemic Injury}

As in our previous study, neovascularization was formed after I/R injury. The VEGF signaling pathway plays a crucial role in angiogenesis. VEGF stimulates cellular responses by binding to VEGFR2, leading to dimerization, and becomes activated through transphosphorylation. That is why we initially determined VEGFR2 expression by Western blotting after harvest- ing tissues. Figure 3a shows that VEGFR2 was somewhat expressed in sham-operated animals; however, its expression level was significantly increased at 4 and $24 \mathrm{~h}$ after I/R injury compared to control. To further investigate whether the VEGFR2 and VEGF mRNA levels were changed, we used quantitative real-time PCR to detect them and found that both VEGF and VEGFR2 mRNA expression were increased at 4 and $24 \mathrm{~h}$ after I/R injury compared to the sham controls $(\mathrm{p}<0.05)$. Although the VEGF expression level at $24 \mathrm{~h}$ was a little lower than at $4 \mathrm{~h}$, its expression level was still higher than that in the control group. These results display that the VEGF signaling pathway may be involved in angiogenesis after I/R injury even though we do not demonstrate that the VEGF receptor VEGFR2 is activated by phosphorylation. 


\section{Microarray Analysis of miRNA Expression during} Renal I/R Injury

Previous studies have proven that miRNAs regulate the VEGF signaling pathway under hypoxia [24]. The question is whether the changes of VEGF and VEGFR2 expression are related to miRNAs after I/R. This prompted us to measure miRNA expression under the condition of injury. We performed microarray analysis and identified miRNA changes after $45 \mathrm{~min}$ of global ischemia followed by $24 \mathrm{~h}$ of reperfusion. Totally, we found that 76 microRNAs exhibiting differences of more than 2-fold were identified in the kidney I/R injury as listed in table 1. Among them, 40 miRNAs were downregulated and 36 miRNAs were upregulated.

Furthermore, we used quantitative real-time PCR to confirm the representative miRNA expression changes which were associated with renal angiogenesis from our microarray analysis. As shown in figure 4, miR-210 expression after I/R injury was increased 2- and 6-fold at 4 and $24 \mathrm{~h}$, respectively. These results are consistent with other reports which showed that miR-210 was upregulated after brain ischemia in rats [25], indicating that miRNAs also play an important role in high-stress conditions.

\section{miR-210 Overexpression Promotes Angiogenesis in vitro}

Combining miR-210 expression with the changes of the VEGF signaling pathway in vivo, we hypothesized that miR-210 may regulate the VEGF pathway and be involved in angiogenesis after renal I/R injury. To this purpose, we infected viral supernatant containing miR-210 into HUVEC-12 cells and made a stable cell line which consistently expresses miR-210. As shown in figure $5 \mathrm{a}$, $100 \%$ of cells expressed miR-210 with GFP. To further confirm miR-210 expression, we extracted mRNA and performed qPCR. The result showed miR-210 was expressed more than 30-fold higher than that in the control cells (fig. 5b).

Other studies indicated that endothelial cells have the ability to form tube-like structures [26]. We followed the same principle to determine whether the HUVEC-12 cell line overexpressing miR-210 can form tube-like structures. From figure 5 c, we can see that cells with miR-210 clearly formed tube-like structures in the Matrigel, unlike those in the control group. Next, we counted tube numbers per field, displaying that there are more than 3 times as many tubes in the miR-210 upregulating group than in the control (fig. 5d). These findings demonstrate that miR-210 plays an important role in angiogenesis.

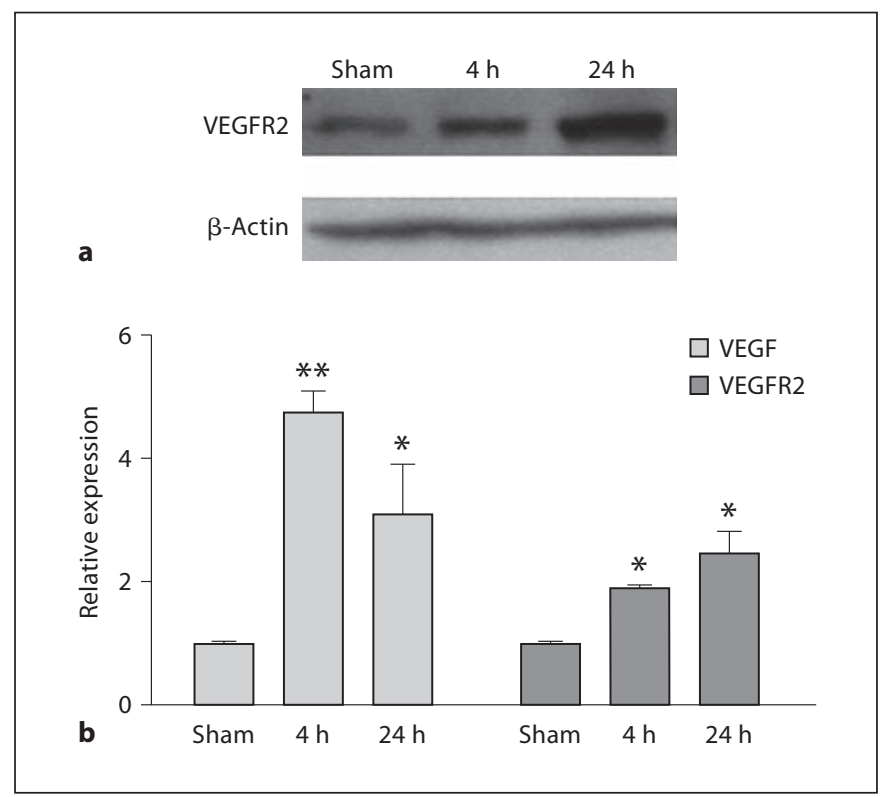

Fig. 3. Expression of VEGF and VEGFR2 after renal I/R injury. a Western blotting for VEGFR2. At 4 and $24 \mathrm{~h}$ after I/R injury, we extracted total protein, ran SDS-PAGE, transferred to a NC membrane and probed with a VEGFR2 antibody. $\beta$-Actin was used as a loading control. b Quantitative real-time PCR for VEGF and VEGFR2 expression. Total RNA was extracted and synthesized to cDNA and quantified by PCR with SYBR and used as an internal control. Both VEGF and VEGFR2 mRNA expression were increased in I/R injury at 4 and $24 \mathrm{~h}$ compared to the sham controls. ${ }^{*} \mathrm{p}<0.05,{ }^{* *} \mathrm{p}<0.01$ vs. sham control.

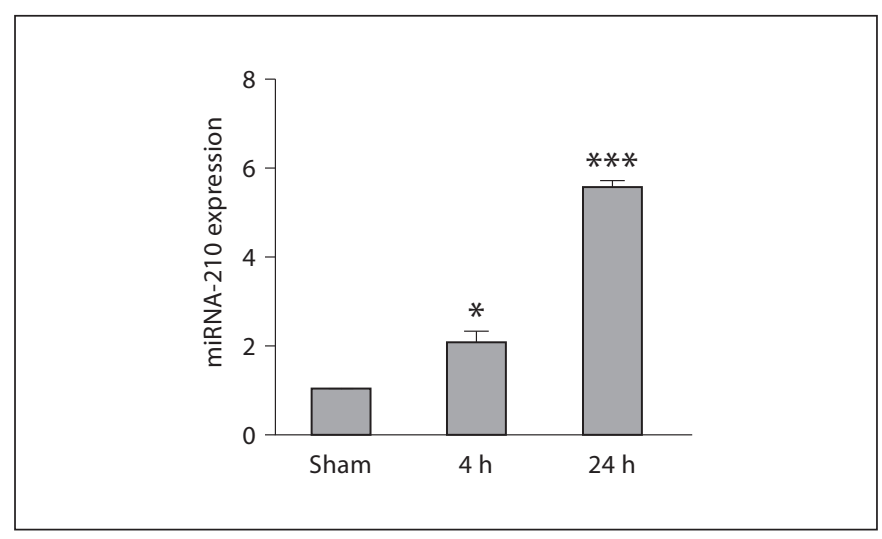

Fig. 4. Expression of miR-210 following renal I/R injury. Quantitative real-time RT-PCR was used to confirm miR-210 expression which was associated with renal angiogenesis from our microarray analysis. miR-210 was upregulated at 4 and $24 \mathrm{~h}$ after I/R injury compared to the control. ${ }^{*} \mathrm{p}<0.05,{ }^{* * *} \mathrm{p}<0.001$ vs. sham control. 
Table 1. A list of microRNAs that showed differential expression in I/R kidneys versus sham kidneys

\begin{tabular}{|c|c|c|c|c|}
\hline & MicroRNA & $\begin{array}{l}\text { Fold } \\
\text { changes } \\
\text { (up- } \\
\text { regulation) }\end{array}$ & MicroRNA & $\begin{array}{l}\text { Fold } \\
\text { changes } \\
\text { (down- } \\
\text { regulation) }\end{array}$ \\
\hline 1 & mmu-miR-21 & 87.85 & mmu-miR-466f-3p & 118.21 \\
\hline 2 & mmu-miR-685 & 73.99 & $\mathrm{mmu}-\mathrm{miR}-466 \mathrm{~g}$ & 81.49 \\
\hline 3 & mmu-miR-876-5p & 54.39 & mmu-miR-669h-3p & 77.35 \\
\hline 4 & mmu-miR-141 & 43.99 & $\mathrm{mmu}-\mathrm{miR}-467 \mathrm{~b}$ & 57.77 \\
\hline 5 & mghv-miR-M1-3 & 41.93 & mmu-miR-467g & 54.41 \\
\hline 6 & mmu-miR-684 & 38.06 & mmu-miR-346 & 44.26 \\
\hline 7 & mmu-miR-218-1 & 26.83 & mmu-miR-669f & 40.99 \\
\hline 8 & mmu-miR-708 & 18.68 & mmu-miR-467e & 34.55 \\
\hline 9 & mmu-miR-302b & 16.83 & mmu-let-7f & 31.60 \\
\hline 10 & mmu-miR-302c & 14.54 & mmu-miR-207 & 30.38 \\
\hline 11 & mmu-miR-466b-5p & 10.85 & mmu-miR-574-3p & 29.89 \\
\hline 12 & $m m u-m i R-532-3 p$ & 9.33 & $\mathrm{mmu}-\mathrm{miR}-877$ & 23.48 \\
\hline 13 & mmu-miR-129-3p & 8.47 & mmu-miR-450a-3p & 17.15 \\
\hline 14 & mmu-miR-483 & 8.26 & mmu-miR-466i & 14.61 \\
\hline 15 & mmu-miR-24-2 & 8.18 & mmu-miR-674 & 14.06 \\
\hline 16 & mghv-miR-M1-8 & 7.17 & $m m u-m i R-466 c-5 p$ & 10.60 \\
\hline 17 & mmu-miR-877 & 6.04 & mmu-miR-328 & 8.30 \\
\hline 18 & mmu-miR-721 & 4.35 & mmu-miR-718 & 8.15 \\
\hline 19 & mmu-miR-126-5p & 3.96 & mmu-miR-212 & 7.14 \\
\hline 20 & mmu-miR-290-3p & 3.82 & mmu-miR-805 & 6.84 \\
\hline 21 & mmu-miR-18a & 3.57 & mmu-miR-483 & 5.70 \\
\hline 22 & $m m u-m i R-671-5 p$ & 3.06 & mmu-miR-181d & 4.71 \\
\hline 23 & mmu-miR-210 & 2.90 & mmu-miR-1196 & 4.53 \\
\hline 24 & mmu-miR-1892 & 2.68 & $m m u-m i R-875-5 p$ & 4.26 \\
\hline 25 & mmu-miR-451 & 2.67 & mmu-miR-467a & 4.25 \\
\hline 26 & mmu-miR-1894-3p & 2.63 & mmu-miR-m01-2 & 3.96 \\
\hline 27 & mmu-miR-705 & 2.60 & mmu-miR-467f & 3.70 \\
\hline 28 & mmu-miR-92a & 2.51 & mmu-miR-30c & 3.58 \\
\hline 29 & mmu-miR-680 & 2.50 & mmu-miR-1198 & 3.52 \\
\hline 30 & mmu-miR-466a-5p & 2.49 & mmu-miR-326 & 3.34 \\
\hline 31 & mmu-miR-188-5p & 2.43 & mmu-miR-200b & 3.34 \\
\hline 32 & mmu-miR-362-5p & 2.30 & $\mathrm{mmu}-\mathrm{miR}-669 \mathrm{a}$ & 3.19 \\
\hline 33 & mmu-miR-30c-1 & 2.29 & mmu-miR-468 & 3.14 \\
\hline 34 & mmu-miR-29c & 2.18 & mmu-miR-211 & 2.91 \\
\hline 35 & mmu-miR-689 & 2.13 & mmu-miR-149 & 2.48 \\
\hline 36 & mmu-miR-19a & 2.00 & mmu-miR-484 & 2.46 \\
\hline 37 & & & mmu-miR-197 & 2.43 \\
\hline 38 & & & mmu-miR-320 & 2.15 \\
\hline 39 & & & mmu-miR-1187 & 2.06 \\
\hline 40 & & & mghv-miR-M1-2 & 2.04 \\
\hline
\end{tabular}

\section{Mechanisms of Angiogenesis under miR-210 Expression}

Since miR-210 expression in endothelial cells promotes the formation of tube-like structures in vitro, we next attempted to define the mechanisms by which miR210 regulates targeted protein expression. To this end, we initially determined VEGF and its receptor VEGFR2 expression in the HUVEC-12 cell line after miR-210 expression. As shown in figure $6 \mathrm{a}$, there is faint expression of both VEGF and VEGFR2 in the control; however, there was stronger expression for both genes in miR-210 expressed cell lines. Meanwhile, we measured the expression of VEGF and VEGFR2 mRNA after miR-210 was upregulated. Figure $6 \mathrm{~b}$ shows that VEGF and VEGFR2 were significantly more expressed in the miR-210 group compared to the control group. Interestingly, VEGF expression increased more than 7 -fold. Taken all together, these findings indicate that the VEGF signaling pathway may be involved in formation of tube-like structures under miR-210 overexpression.

\section{Discussion}

Renal I/R injury stimulated a multifaceted cascade of physiological and biochemical events. It is believed that these events are associated with the interaction of many genes and proteins. Despite decades of research, the cellular and molecular basis of I/R injury remain to be fully understood, and effective therapies of I/R injury are currently lacking.

MiRNAs are small non-coding RNAs and regulate gene expression at the post-transcriptional level by either degradation or translational repression of a target mRNA that regulates physiological and pathological processes. However, very little is known about the role and regulation of miRNAs in renal pathophysiology. We performed miRNA arrays to detect the expression pattern of miRNAs in murine kidneys subjected to I/R injury in vivo. Our study identified 76 miRNAs exhibiting more than 2-fold changes in the kidney I/R injury, of which 40 miRNAs downregulated and 36 miRNAs upregulated. Analysis of the differential miRNA expression profiles in the sham group and renal ischemia group indicated that I/R injury had pleiotropic effects in the kidneys, which alter essential renal functions. To date, only a few reports are available on the miRNA microarray profiling of the renal ischemia [27].

Furthermore, animal experiments and clinical studies [28] showed that compensatory angiogenesis was increased after tissue ischemia. Also, most studies [29, 30] have confirmed that the VEGF signaling pathway plays a critical regulatory role in endothelial cell proliferation, differentiation, and migration and is involved in the regulation of ischemia-induced angiogenesis in tissue ischemia, which is also the most important mechanism in reg- 
Fig. 5. Angiogenesis under miR-210 overexpression in HUVEC-12 cells. a, b miR210 overexpression in HUVEC-12 cells. HUVEC-12 cells were transduced lentivirus containing miR-210 or control and incubated overnight. miR-210 was expressed with GFP in the cells (a). Total RNA was extracted at $72 \mathrm{~h}$ after miR-210 was expressed and real-time PCR was used to double check it. In comparison with the control, there was a more than 30 -fold increase in miR-210 expressed cells. c, d Upregulated miR-210 enhanced neovascularization formation. After HUVEC-12 cells with miR-210 expression were cultured for $24 \mathrm{~h}$, tube-like structures were formed (c). A higher number of tube-like structures per field appeared in miR-210 overexpressed cells than that in control (d). ${ }^{* *} \mathrm{p}<0.01,{ }^{* *} \mathrm{p}<0.001$ vs. control.

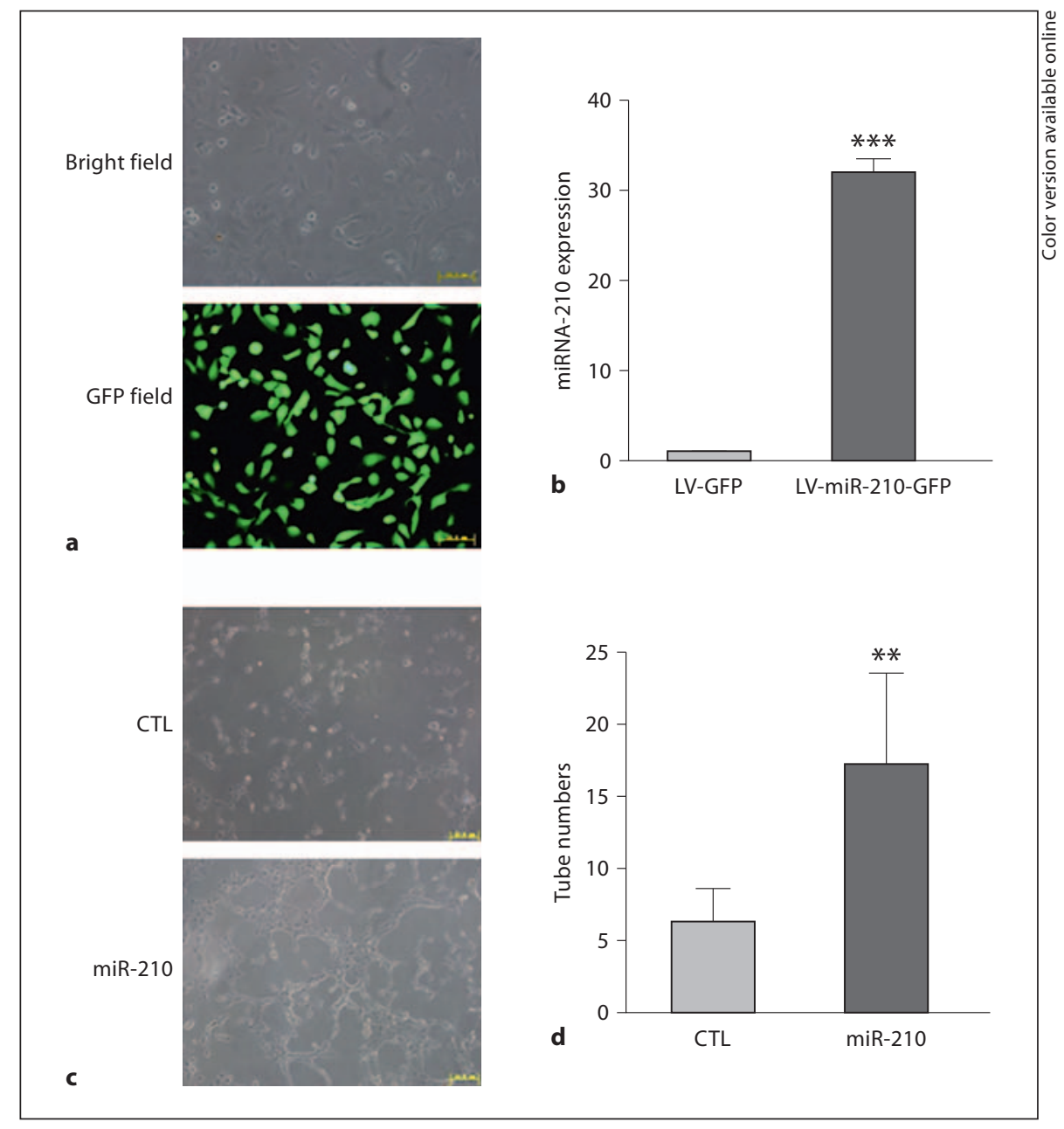

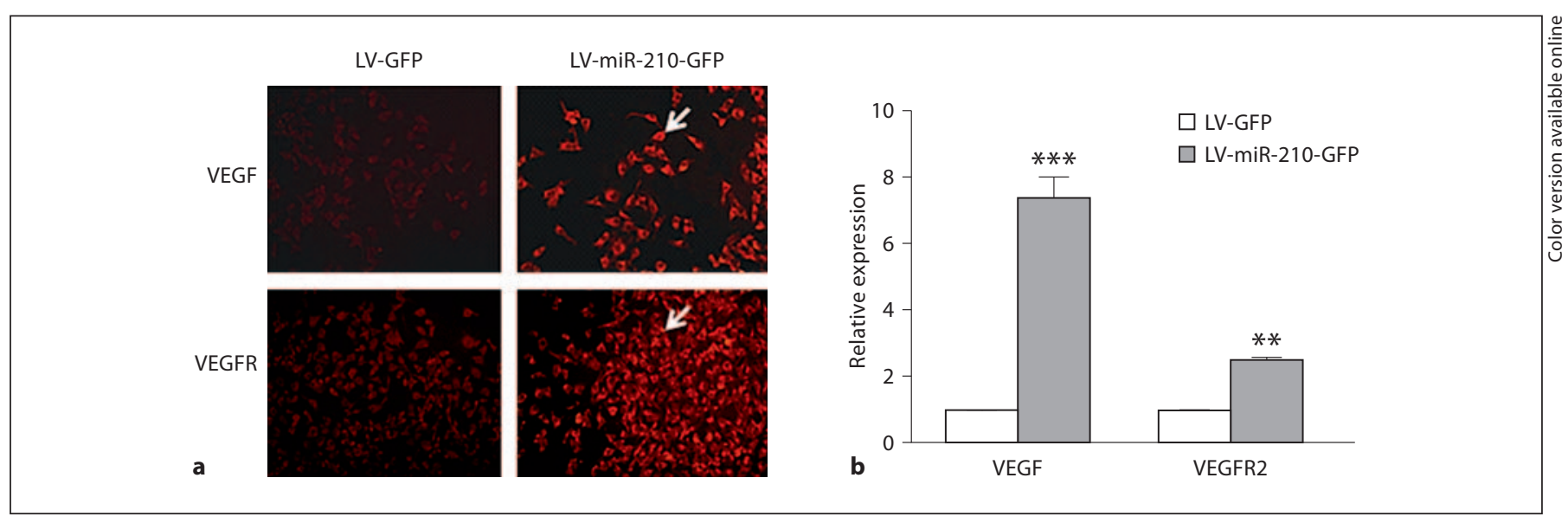

Fig. 6. VEGF and VEGFR2 expression were increased in miR-210 overexpressed cells. a VEGF and VEGFR2 were immunostained. Cells were seeded on coverslips and incubated overnight and fixed with $4 \%$ paraformaldehyde, followed by a standard immunocytochemistry procedure. Expression of both VEGF and its receptor were greater in miR-210 cells than that in the control (arrows). b VEGF and VEGFR2 mRNA expression. mRNA was extracted using TRIzol and expression was quantified by real-time PCR. mRNA expression of VEGF and VEGFR2 showed a significant increased in upregulated miR-210 HUVEC-12 cells. ${ }^{* *} \mathrm{p}<0.01$, *** $\mathrm{p}<0.001$ vs. control. 
ulation of angiogenesis in renal ischemia. VEGF acts via VEGFR2 which triggers a cascade of biochemical events, especially stimulating endothelial cell division, proliferation, migration and even neovascularization. To prove ischemia-induced angiogenesis in renal ischemia, we used the endothelial cell marker CD31 which is expressed on the endothelial cell surface to detect whether angiogenesis was formed in response to ischemic injury. As predicted, the immunohistochemistry staining results of CD31 showed a significant increase of microvessel in the renal ischemic region compared with the control group. VEGF and VEGFR2 mRNA and protein expression were increased in I/R injury compared to the sham controls.

Recently, a few specific miRNAs targeting endothelial cell function and angiogenesis have been identified. Whether miRNAs participate in the angiogenesis mechanisms of renal I/R injury is completely unknown. In our present study, 76 miRNAs significantly changed on the renal I/R injury in our microarray result, some of which were reported to be involved in angiogenesis, such as miR-210. Notably, we used the HUVEC-12 cell line as an in vitro model to study the role of miR-210 on regulating VEGF and VEGFR2 expression even though HUVEC-12 cells may be different from endothelial cells in the capillaries around renal tubules. Our results suggest that miR210 regulates expression of both VEGF and VEGFR2, and is related to angiogenesis and ischemic diseases.

Hua et al. [24] suggested that miRNAs directly regulated expression of VEGF and other angiogenic factors. Through computational analysis, they found that miR210 is a putative regulator of VEGF and could be involved in VEGF signaling regulation. MiR-210 was upregulated in response to hypoxia in vitro and in vivo inhibiting the target genes of ephrin-A3 protein expression and stimulating capillary-like formation [31]. Ephrin-A3, as an eph- rin ligand family member, can join the Eph/ephrin system to be controlled by the VEGF pathway regulating angiogenesis [32, 33]. Recently, Bonauer et al. [9] identified that miR-92a may serve as a valuable therapeutic target in the setting of ischemic diseases. They found that overexpression of miR-92a in endothelial cells blocked angiogenesis in vitro. Inhibiting miR-92a by antagomir-92a treatment in mice can promote neovascularization and increase the expression of target ITGA 5 mRNA and protein in the vasculature of skeletal muscle tissue. Meanwhile, ITGA5 was the integrin family member which was a promoted angiogenesis factor to be stimulated by the VEGF regulating angiogenesis $[34,35]$. In our microarray data, we also observed that ischemic renal injury significantly increased miR-92a expression compared to the sham-operated control with 2 -fold changes (table 1). In future studies, we should further confirm that miR-92a function to suppress angiogenesis by overexpressing miR-92a in HUVEC-12 cells.

In summary, our results imply that miR-210 might be important miRNAs directly associated with the VEGF signal pathway relevant to angiogenesis in renal ischemia, thus strengthening the hypothesis of the role of microRNAs in renal angiogensis. The specific miRNAs, as key regulators of the response to renal ischemia, have provided novel insights into the angiogenesis mechanism of renal I/R injury and opened clinical avenues. Further studies are required to understand the mechanisms of miRNA-based angiogenesis.

\section{Acknowledgments}

This work was supported by National Natural Science Foundation of China (grant Nos. 30960385, 3096039 and 810600596).

\section{References}

1 Basile DP: The endothelial cell in ischemic acute kidney injury: implications for acute and chronic function. Kidney Int 2007;72: 151-156.

-2 Freitas MC, Uchida Y, Zhao D, Ke B, Busuttil RW, Kupiec-Weglinski JW: Blockade of Janus kinase-2 signaling ameliorates mouse liver damage due to ischemia and reperfusion. Liver Transpl 2010;16:600-610.

3 Wasserberg N, Pileggi A, Salgar SK, Ruiz P, Ricordi C, Inverardi L, Tzakis AG: Heme oxygenase-1 upregulation protects against intestinal ischemia/reperfusion injury: a laboratory based study. Int J Surg 2007;5:216-224.
4 Shibuya M: Vascular endothelial growth factor-dependent and -independent regulation of angiogenesis. BMB Rep 2008;41:278-286.

5 Vincent KA, Shyu KG, Luo Y, Magner M, Tio RA, Jiang C, Goldberg MA, Akita GY, Gregory RJ, Isner JM: Angiogenesis is induced in a rabbit model of hindlimb ischemia by naked DNA encoding an HIF-1 $\alpha /$ VP16 hybrid transcription factor. Circulation 2000;102: 2255-2261.

-6 Shibuya M, Claesson-Welsh L: Signal transduction by VEGF receptors in regulation of angiogenesis and lymphangiogenesis. Exp Cell Res 2006;312:549-560.
7 Ren XP, Wu J, Wang X, Sartor MA, Qian J, Jones K, Nicolaou P, Pritchard TJ, Fan GC: MicroRNA-320 is involved in the regulation of cardiac ischemia/reperfusion injury by targeting heat-shock protein 20. Circulation 2009;119:2357-2366.

8 Kuehbacher A, Urbich C, Zeiher AM, Dimmeler S: Role of Dicer and Drosha for endothelial microRNA expression and angiogenesis. Circ Res 2007;101:59-68. 
-9 Bonauer A, Carmona G, Iwasaki M, Mione M, Koyanagi M, Fischer A, Burchfield J, Fox $\mathrm{H}$, Doebele C, Ohtani K, Chavakis E, Potente M, Tjwa M, Urbich C, Zeiher AM, Dimmeler S: Microrna-92a controls angiogenesis and functional recovery of ischemic tissues in mice. Science 2009;324:1710-1713.

- 10 Fish JE, Santoro MM, Morton SU, Yu S, Yeh RF, Wythe JD, Ivey KN, Bruneau BG, Stainier DY, Srivastava D: miR-126 regulates angiogenic signaling and vascular integrity. Dev Cell 2008;15:272-284.

11 Ambros V: The functions of animal micro RNAs. Nature 2004;431:350-355.

-12 He L, Hannon GJ: MicroRNAs: small RNAs with a big role in gene regulation. Nat Rev Genet 2004;5:522-531.

-13 Kato M, Arce L, Natarajan R: MicroRNAs and their role in progressive kidney diseases. Clin J Am Soc Nephrol 2009;4:1255-1266.

-14 Roy S, Khanna S, Hussain SR, Biswas S, Azad A, Rink C, Gnyawali S, Shilo S, Nuovo GJ, Sen CK: MicroRNA expression in response to murine myocardial infarction: miR-21 regulates fibroblast metalloprotease- 2 via phosphatase and tensin homologue. Cardiovasc Res 2009;82:21-29.

-15 Yuan Y, Wang JY, Xu LY, Cai R, Chen Z, Luo BY: MicroRNA expression changes in the hippocampi of rats subjected to global ischemia. J Clin Neurosci 2010;17:774-778.

16 Xu CF, Yu CH, Li YM: Regulation of hepatic microRNA expression in response to ischemic preconditioning following ischemia/ reperfusion injury in mice. OMICS 2009;13: 513-520.

17 Pulkkinen K, Malm T, Turunen M, Koistinaho J, Yla-Herttuala S: Hypoxia induces microRNA miR-210 in vitro and in vivo ephrin-A3 and neuronal pentraxin-1 are potentially regulated by miR-210. FEBS Lett 2008; 582:2397-2401.
18 Brooks C, Wei Q, Cho SG, Dong Z: Regulation of mitochondrial dynamics in acute kidney injury in cell culture and rodent models. J Clin Invest 2009;119:1275-1285.

19 Wei Q, Yin XM, Wang MH, Dong Z: Bid deficiency ameliorates ischemic renal failure and delays animal death in C57BL/6 mice. Am J Physiol Renal Physiol 2006;290:F35F42.

20 El Nahas AM, Bassett AH, Cope GH, Le Carpentier JE: Role of growth hormone in the development of experimental renal scarring. Kidney Int 1991;40:29-34.

-21 Hugo C, Pichler R, Meek R, Gordon K, Kyriakides T, Floege J, Bornstein P, Couser WG, Johnson RJ: Thrombospondin-1 is expressed by proliferating mesangial cells and is up-regulated by PDGF and bFGF in vivo. Kidney Int 1995;48:1846-1856.

22 Hohenstein B, Braun A, Amann KU, Johnson RJ, Hugo CP: A murine model of sitespecific renal microvascular endothelial injury and thrombotic microangiopathy. Nephrol Dial Transplant 2008;23:11441156.

23 Chen C, Ridzon DA, Broomer AJ, Zhou Z, Lee DH, Nguyen JT, Barbisin M, Xu NL, Mahuvakar VR, Andersen MR, Lao KQ, Livak KJ, Guegler KJ: Real-time quantification of microRNAs by stem-loop RT-PCR. Nucleic Acids Res 2005;33:e179.

24 Hua Z, Lv Q, Ye W, Wong CK, Cai G, Gu D, Ji Y, Zhao C, Wang J, Yang BB, Zhang Y: MiRNA-directed regulation of VEGF and other angiogenic factors under hypoxia. PLoS One 2006; 1:e116.

25 Pulkkinen K, Malm T, Turunen M, Koistinaho J, Ylä-Herttuala S: Hypoxia induces microRNA miR-210 in vitro and in vivo ephrin-A 3 and neuronal pentraxin-1 are potentially regulated by miR-210. FEBS Lett 2008; 582:2397-2401.

-26 Sekiya S, Muraoka M, Sasagawa T, Shimizu T, Yamato M, Okano T: Three-dimensional cell-dense constructs containing endothelial cell-networks are an effective tool for in vivo and in vitro vascular biology research. $\mathrm{Mi}$ crovasc Res 2010;80:549-551.
27 Wei Q, Bhatt K, He HZ, Mi QS, Haase VH, Dong Z: Targeted deletion of Dicer from proximal tubules protects against renal ischemia-reperfusion injury. J Am Soc Nephrol 2010;21:756-761

28 Lerman LO, Chade AR: Angiogenesis in the kidney: a new therapeutic target? Curr Opin Nephrol Hypertens 2009;18:160-165.

$>29$ Kroll J, Waltenberger J: The vascular endothelial growth factor receptor KDR activates multiple signal transduction pathways in porcine aortic endothelial cells. J Biol Chem 1997;272:32521-32527.

>30 Waltenberger J, Claesson-Welsh L, Siegbahn A, Shibuya M, Heldin CH: Different signal transduction properties of KDR and Flt1, two receptors for vascular endothelial growth factor. J Biol Chem 1994;269:2698826995.

-31 Fasanaro P, D’Alessandra Y, Di Stefano V, Melchionna R, Romani S, Pompilio G, Capogrossi MC, Martelli F: MicroRNA-210 modulates endothelial cell response to hypoxia and inhibits the receptor tyrosine kinase ligand ephrin-A3. J Biol Chem 2008;283: 15878-15883.

32 Bhuvaneswari R, Gan YY, Lucky SS, Chin WW, Ali SM, Soo KC, Olivo M: Molecular profiling of angiogenesis in hypericin-mediated photodynamic therapy. Mol Cancer 2008;7:56.

-33 Kuijper S, Turner CJ, Adams RH: Regulation of angiogenesis by Eph-ephrin interactions. Trends Cardiovasc Med 2007;17:145-151.

\34 Milner R, Hung S, Erokwu B, Dore-Duffy P, LaManna JC, del Zoppo GJ: Increased expression of fibronectin and the $\alpha_{5} \beta_{1}$ integrin in angiogenic cerebral blood vessels of mice subject to hypobaric hypoxia. Mol Cell Neurosci 2008;38:43-52.

35 Garmy-Susini B, Varner JA: Roles of integrins in tumor angiogenesis and lymphangiogenesis. Lymphat Res Biol 2008;6:155163. 